\title{
Perfil epidemiológico de pacientes acometidos por leptospirose em um estado brasileiro na Amazônia Ocidental
}

\author{
Alesandro Lima Rodrigues \\ Enfermeiro pela Faculdade Barão do Rio Branco (FAB). \\ Especialista em Saúde da Família pela Universidade Candido Mendes (UCAM), Rio de Janeiro, Brasil. \\ 凶alesandrorodrigues.ac1@gmail.com
}

Recebido em 1 de fevereiro de 2019

Aceito em 16 de maio de 2019

\begin{abstract}
Resumo:
Introdução: A leptospirose é causada pela bactéria Lepstospira, fazendo parte do grupo das doenças de notificação compulsória. No país, a leptospirose é um problema de saúde pública que envolve vários fatores e consequências. Objetivo: Este estudo teve por objetivo identificar o perfil epidemiológico de pacientes acometidos por leptospirose no estado do Acre-Amazônia Ocidental, no ano de 2017. Metodologia: Estudo de caráter epidemiológico-descritivo, de abordagem quantitativa sobre os casos confirmados de leptospirose no estado do Acre no ano de 2017. Os dados foram coletados online no Sistema de Informação de Agravos de Notificação (SINAN). Resultados: Após análise dos dados, constatou-se que o perfil epidemiológico da doença no estado do Acre, foi prevalente no sexo masculino $(66,5 \%)$, com maior número de casos na faixa etária de 20-39 anos $(38,8 \%)$ e com nível fundamental incompleto $(35,9 \%)$. Também foi possível verificar que mais da metade dos casos foram na zona urbana (69,9\%), nas cidades de Rio Branco $(44,5 \%)$ e Cruzeiro do Sul (37,8\%). Quanto ao desfecho clínico, verificou-se um elevado percentual de indivíduos curados $(96,1 \%)$ e baixa letalidade, em que apenas $(1 \%)$ dos casos foram a óbito devido à doença. Conclusão: Assim, conclui-se que a aplicação de atitudes pessoais de prevenção e no dever da administração pública de proporcionar saneamento básico, educação em saúde e infraestrutura ao indivíduo, os subsídios necessários para o enfrentamento da leptospirose.
\end{abstract}

Palavras-chave: Vigilância epidemiológica, Leptospirose Humana, Perfil epidemiológico.

\section{Epidemiological profile of patients affected by leptospirosis in a Brazilian state in the Western Amazon}

\begin{abstract}
:
Introduction: Leptospirosis is caused by the bacterium Leptospira, being part of the group of diseases of compulsory notification. In the country, leptospirosis is a public health problem that involves several factors and consequences. Objective: The objective of this study was to identify the epidemiological profile of patients with leptospirosis in the state of Acre-Amazon western in the year 2017. Methodology: Epidemiological descriptive study of a quantitative approach on confirmed cases of leptospirosis in the state of Acre in the year 2017. The data were collected online in the Notification diseases Information System (SINAN). Results: After analyzing the data, it was verified that the epidemiological profile of the disease in the state of Acre, was prevalent in males (66.5\%), with the highest number of cases in the age group of 20-39 years (38.8\%) and with incomplete fundamental level (35.9\%). It was also possible to verify that more than half of the cases were in the urban area (69.9\%), in the cities of Rio Branco (44.5\%) and Cruzeiro do Sul (37.8\%). Regarding the clinical outcome, there was a high percentage of cured individuals (96.1\%) and low lethality, in which only (1\%) of the cases were death due to the disease. Conclusion: Thus, it is concluded that the
\end{abstract}


application of personal preventive attitudes and the duty of the public administration to provide basic sanitation, health education and infrastructure to the individual, the necessary subsidies for coping with leptospirosis.

Keywords: Epidemiological surveillance, Human Leptospirosis, Epidemiological profile.

\section{Perfil epidemiológico de pacientes afectados por leptospirosis en un estado brasileño en la Amazonía Occidental}

\section{Resumen:}

Introducción: La leptospirosis es causada por la bacteria Lepstospira, formando parte del grupo de las enfermedades de notificación obligatoria. En el país, la leptospirosis es un problema de salud pública que involucra varios factores y consecuencias. Objetivo: Este estudio tuvo por objetivo identificar el perfil epidemiológico de pacientes afectados por leptospirosis en el estado de Acre-Amazonia Occidental, en el año 2017. Metodología: Estudio de carácter epidemiológico-descriptivo de abordaje cuantitativo sobre los casos confirmados de leptospirosis en el estado de Acre en el año 2017. Los datos fueron recolectados online en el Sistema de Información de Agravios de Notificación (SINAN). Resultados: Después del análisis de los datos, se constató que el perfil epidemiológico de la enfermedad en el estado de Acre, fue predominante en el sexo masculino (66,5\%), con mayor número de casos en el grupo de edad de $20-39$ años $(38,8 \%)$ y con un nivel fundamental incompleto $(35,9 \%)$. También fue posible verificar que más de la mitad de los casos fueron en la zona urbana $(69,9 \%)$, en las ciudades de Rio Branco $(44,5 \%)$ y Cruzeiro do Sul $(37,8 \%)$. En cuanto al desenlace clínico, se verificó un elevado porcentaje de individuos curados $(96,1 \%)$ y baja letalidad, en la que sólo (1\%) de los casos fueron la muerte debido a la enfermedad. Conclusión: Así, se concluye que la aplicación de actitudes personales de prevención y en el deber de la administración pública de proporcionar saneamiento básico, educación en salud e infraestructura al individuo, los subsidios necesarios para el enfrentamiento de la leptospirosis.

Palabras clave: Vigilancia epidemiológica, Leptospirosis humana, Perfil epidemiológico.

\section{INTRODUÇÃo}

A leptospirose é uma doença zoonótica de distribuição global, provocada por bactérias do gênero Leptospira, que infectam animais silvestres, sinantrópicos, domésticos e o homem (BORGHI; QUEIROZ, 2017). Sua ocorrência é prevalente nas regiões de clima tropical e subtropical, principalmente na América Latina e Caribe (SIMÕES et al., 2016).

Um estudo internacional realizado por pesquisadores da Fundação Oswaldo Cruz Bahia em parceria com a Universidade Federal da Bahia, Escola de Saúde Pública de Yale, Universidade de Zurique e a Organização Mundial de Saúde, apontou que a carga global da leptospirose causará mais de um milhão de novas infecções e cerca de 59 mil óbitos por ano no mundo (COSTA et al., 2015).

No Brasil, de 2010 a 2014, foram confirmados 20.810 casos de leptospirose, dos quais 
1.694 foram a óbito. O maior número de casos foi observado nas regiões Sudeste (7.457) e Sul (6.030), seguidos pelas regiões Norte (3.929), Nordeste (3.141) e Centro-Oeste (253). Os estados com maior percentual de casos confirmados foram: São Paulo (20,9\%), Santa Catarina (10,7\%), Rio Grande do Sul (10,6\%) e Acre (10,5\%) (BRASIL, 2016).

Em geral, no país, os casos aumentam no período de verão - caráter sazonal resultante das chuvas e alagamentos nas áreas urbanas (FONZAR, 2010). Além da questão pluviométrica, mantém relação com as precárias condições de moradia e alta infestação de roedores infectados (CASTRO et al., 2010).

Na zona urbana, os roedores são o principal reservatório e disseminador da bactéria que ocorre através da urina. Ao entrar em contato com a água, as bactérias ficam livres no ambiente e podem infectar o homem (AVILA-PIRES, 2006). De modo geral, o ser humano se contamina por meio da urina de animais infectados, da água de enchente/esgoto ou alimentos contaminados pelas bactérias (DE SOUZA, 2011). Quanto ao contato, o microrganismo pode penetrar por meio de lesões na pele, mucosa ou mesmo na pele integra (BRASIL, 2014).

Ao entrar no organismo humano, a bactéria desenvolve a doença da leptospirose, que causa várias manifestações sistêmicas (DAHER et al., 2003). Entre os principais sintomas estão tosse, febre, vômito, dor de cabeça, diarreia e dor no corpo (BRASIL, 2017a). O indivíduo ainda pode apresentar quadros graves da doença, como anemia grave, arritmias, miocardite, pancreatite, insuficiência renal aguda, necrose tubular aguda, meningite asséptica, convulsões, encefalite, síndrome de Weil e de Guillain-Barré (BRASIL, 2017b).

O diagnóstico da leptospirose é realizado por meio da análise da história clínica e dos testes específicos-laboratoriais como o teste ELISA-IgM e a microaglutinação-MAT. O tratamento ocorre através do uso terapêutico de antibióticos e reidratação venosa; na situação grave é estabelecido a penicilina G cristalina, ampicilina e ceftriaxona, e no caso leve, amoxicilina, azitromicina e doxiciclina (BRASIL, 2009).

De acordo com Chaiblich et al. (2017), a leptospirose é uma doença pertinente para área da vigilância epidemiológica e da saúde pública, devido sua forma de disseminação e de suas consequências no organismo, demonstrando ser um grave problema da questão da infraestrutura socioambiental no país. 
Diante do exposto, o presente estudo objetiva identificar o perfil epidemiológico de pacientes acometidos por leptospirose no estado do Acre - Amazônia Ocidental, no ano de 2017.

\section{METODOLOGIA}

Estudo de caráter epidemiológico, retrospectivo, descritivo, de abordagem quantitativa sobre os casos confirmados de leptospirose no estado do Acre no ano de 2017, notificados no Sistema de Informação de Agravos de Notificação (SINAN), na base de dados do departamento de informática do Sistema único de Saúde (DATASUS-TABNET).

Os casos suspeitos de leptospirose são aqueles em que o indivíduo apresenta febre, cefaleia, mialgia associada a pelo menos um destes sinais: aumento da bilirrubina, icterícia, insuficiência renal ou que tenha sido exposto à água ou lama de inundação nos últimos 30 dias de início dos sintomas. Os casos confirmados, por sua vez, são aqueles em que o indivíduo apresenta os mesmos sinais e sintomas de casos suspeitos, mas com resultados de testes laboratoriais-específicos reagentes para Leptospira (GOVERNO DO ESTADO DA BAHIA, 2017).

A população do presente estudo é composta por todos os casos confirmados de leptospirose no estado do Acre no ano de 2017. Para definir o perfil epidemiológico da doença, os dados foram selecionados de acordo com as seguintes variáveis: sexo, faixa etária, escolaridade, município de infecção/caracterização da zona e evolução clínica dos acometidos.

Os dados obtidos foram analisados através dos métodos estatísticos de medidas de frequência absoluta e percentual e tabulados por meio do programa Microsoft Excel 2013 com apresentação em forma de gráficos e tabelas.

Por se tratar de dados secundários de domínio público e de livre acesso no Sistema DATASUS, não houve necessidade desse estudo ser submetido à apreciação do Comitê de Ética em Pesquisa (CEP) da Comissão Nacional de Ética em Pesquisa (CONEP). 


\section{RESULTADOS}

A Amazônia ocidental é constituída pelos estados do Acre, Amazonas, Rondônia e Roraima (SUFRAMA, 2017). Em 2017, foram notificados 308 casos confirmados de leptospirose nesses quatro estados por meio do Sistema de Informação de Agravos de Notificação (SINAN). Destes, 209 casos de leptospirose ocorreram no estado do Acre.

A Figura 1 apresenta a distribuição dos casos de leptospirose no estado do Acre no ano de 2017, segundo o sexo. Observa-se que entre os pacientes acometidos, que a maior frequência de casos foi no sexo masculino 139 (66,5\%) e com um menor percentual o sexo feminino $70(33,5 \%)$.

Figura 1 - Distribuição dos casos de leptospirose notificados no Estado do Acre no ano de 2017, segundo o sexo.

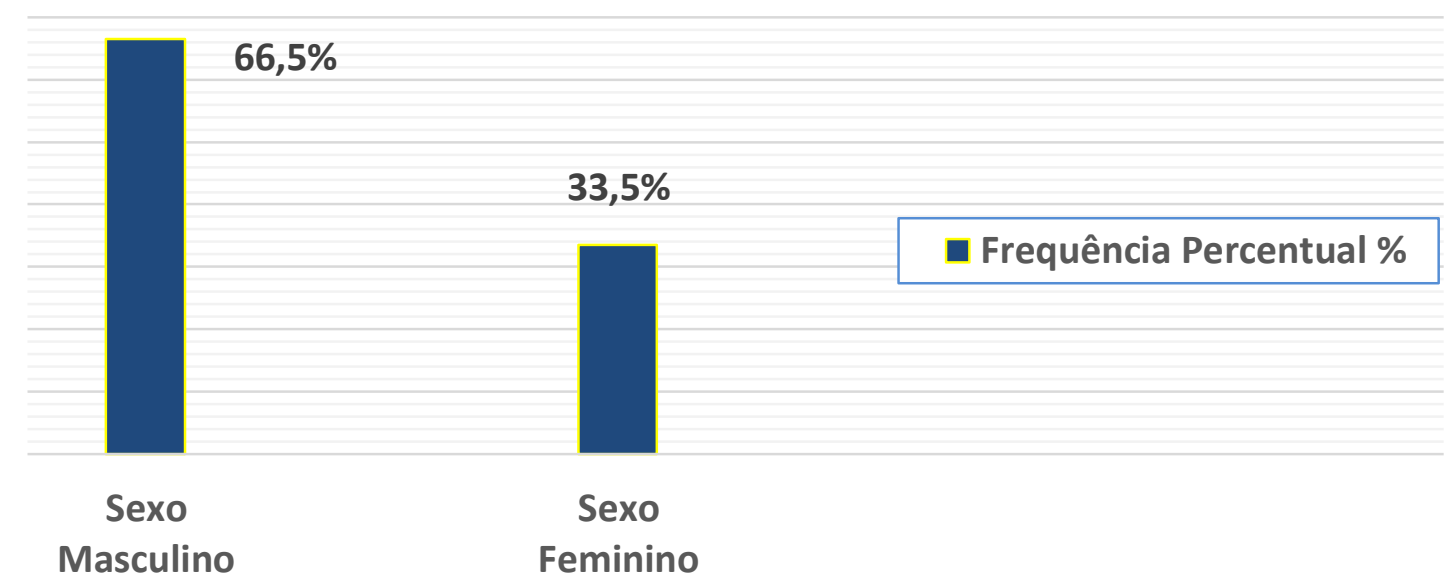

Fonte de Dados: Ministério da Saúde - Sistema de Informação de Agravos de Notificação - Sinan Net.

A Figura 2 mostra a classificação da faixa etária dos pacientes com leptospirose, independente do Sexo. Verifica-se que a maioria dos pacientes estavam na faixa etária de 20-39 anos (38,8\%), 40-59 anos (32\%) e a minoria entre menores de 01 ano a 19 anos $(23,5 \%)$ e de 60-79 anos (5,7\%). 
Figura 2 - Distribuição dos casos de leptospirose notificados no Estado do Acre, no ano de 2017, segundo a faixa etária.

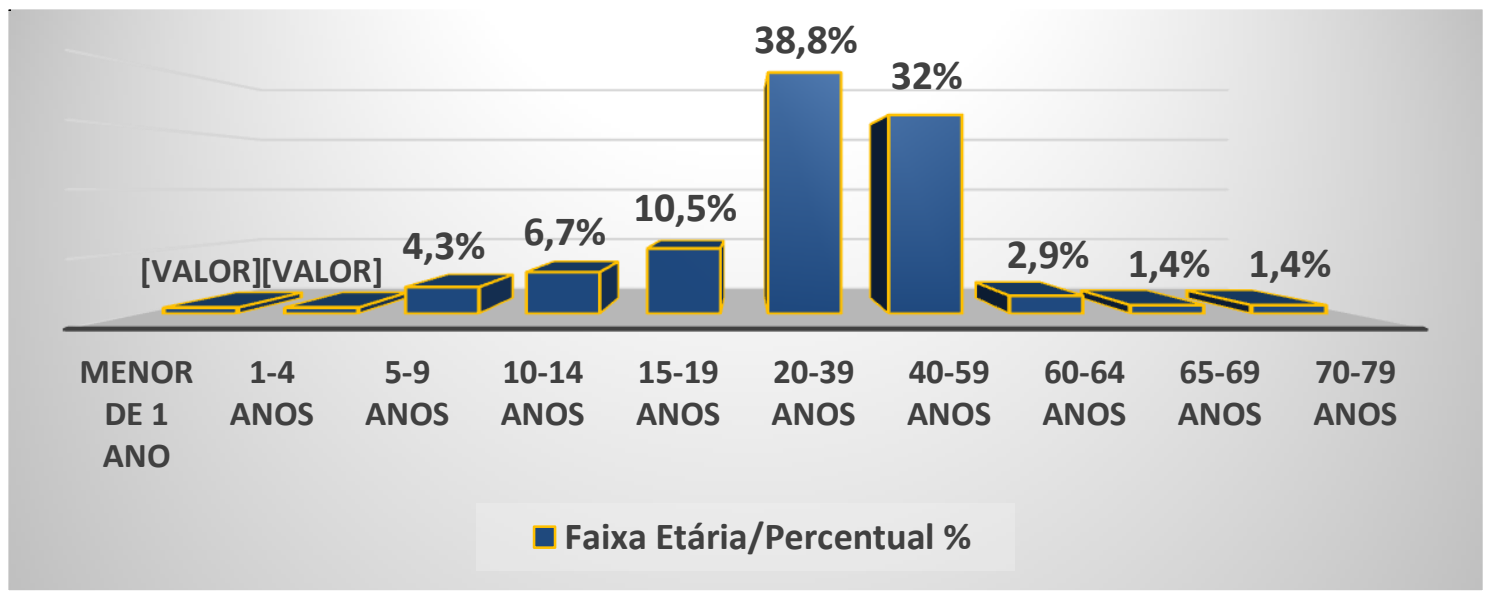

Fonte de Dados: Ministério da Saúde - Sistema de informação de Agravos de Notificação - Sinan Net.

A Figura 3 expõe o nível de escolaridade dos pacientes acometidos por leptospirose, independentemente do sexo. Observa-se que as maiores parcelas dos casos notificados no SINAN Net - secção Acre, foram em pacientes com ensino fundamental incompleto 75 (35,9\%) e nível escolar ignorado/branco 40 (19,1\%). Baixo percentual da doença ocorreu em indivíduos com ensino superior incompleto 2 (1\%) e completo 2 (1\%) dos casos confirmados.

Figura 3 - Distribuição dos casos de leptospirose notificados no Estado do Acre, no ano de 2017, segundo o nível de escolaridade.

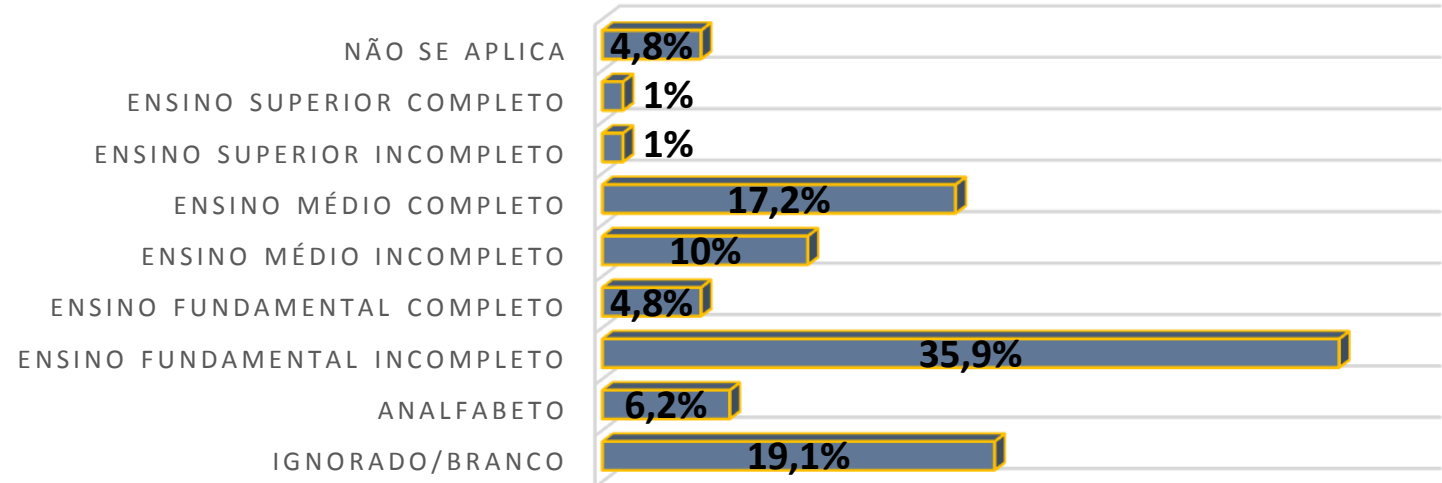

Fonte de Dados: Ministério da Saúde - Sistema de informação de Agravos de Notificação - Sinan Net 
A Tabela 1 apresenta os municípios de infecção e a caracterização da zona/área dos pacientes com diagnóstico confirmado de leptospirose. Verifica-se que a maior proporção de notificação da doença ocorreu na zona urbana 146 (69,9\%), e os municípios de residência com elevados números de casos foram em Rio Branco-AC 93 (44,5\%) e Cruzeiro do Sul-AC 79 $(37,8 \%)$.

Tabela 1 - Distribuição dos casos de leptospirose notificados no Estado do Acre, no ano de 2017, segundo o município de infecção e a classificação da área.

\begin{tabular}{|c|c|c|}
\hline ZONA & FREQUÊNCIA ABSOLUTA & FREQUÊNCIA PERCENTUAL \\
\hline Ignorado/Branco & 09 & $4,3 \%$ \\
\hline Urbana & 146 & $69,9 \%$ \\
\hline Rural & 49 & $23,4 \%$ \\
\hline Periurbana & 05 & $2,4 \%$ \\
\hline TOTAL & 209 casos & $100 \%$ \\
\hline MUNICÍPIO & FREQUÊNCIA ABSOLUTA & FREQUÊNCIA PERCENTUAL \\
\hline Acrelândia & 3 & $1,4 \%$ \\
\hline Brasiléia & 2 & $1 \%$ \\
\hline Bujari & 7 & $3,3 \%$ \\
\hline Capixaba & 1 & $0,5 \%$ \\
\hline Cruzeiro do Sul & 79 & $37,8 \%$ \\
\hline Feijó & 7 & $3,3 \%$ \\
\hline Mâncio Lima & 3 & $1,4 \%$ \\
\hline Marechal Thaumaturgo & 1 & $0,5 \%$ \\
\hline Plácido de Castro & 1 & $0,5 \%$ \\
\hline Porto Acre & 3 & $1,4 \%$ \\
\hline Porto Walter & 1 & $0,5 \%$ \\
\hline Rio Branco & 93 & $44,5 \%$ \\
\hline Rodrigues Alves & 1 & $0,5 \%$ \\
\hline Santa Rosa do Purus & 1 & $0,5 \%$ \\
\hline Sena Madureira & 3 & $1,4 \%$ \\
\hline Senador Guiomard & 2 & $1 \%$ \\
\hline Xapuri & 1 & $0,5 \%$ \\
\hline TOTAL & 209 casos & $100 \%$ \\
\hline
\end{tabular}

Fonte de Dados: Ministério da Saúde - Sistema de informação de Agravos de Notificação - Sinan Net. 
A Tabela 2 mostra a evolução e o desfecho clínico dos casos de leptospirose no Acre segundo o sexo. Constata-se que a proporção de indivíduos que obtiveram cura foi alta 201 $(96,1 \%)$ dos casos, 05 (2,4\%) tiveram o desfecho ignorado/branco e apenas 02 (1\%) foram a óbito pelo agravo notificado.

Tabela 2 - Evolução clínica e desfecho dos casos notificados de leptospirose no Estado do Acre em 2017, segundo o sexo.

\begin{tabular}{l|ccc}
\multicolumn{1}{c}{ EVOLUÇÃO } & $\begin{array}{r}\text { FREQUÊNCIA } \\
\text { ABSOLUTA }\end{array}$ & $\begin{array}{c}\text { FREQUÊNCIA } \\
\text { PERCENTUAL }\end{array}$ & SEXO \\
\hline IGNORADO/BRANCO & 05 & $2,4 \%$ & Masculino \\
CURA & 201 & $96,1 \%$ & Masculino/Feminino \\
$\begin{array}{l}\text { ÓBITO PELO AGRAVO } \\
\text { NOTIFICADO }\end{array}$ & 02 & $1 \%$ & Masculino \\
$\begin{array}{l}\text { ÓBITO POR OUTRA } \\
\text { CAUSA }\end{array}$ & 01 & $0,5 \%$ & Masculino \\
\end{tabular}

Fonte de Dados: Ministério da Saúde - Sistema de informação de Agravos de Notificação - Sinan Net

\section{DISCUSSÃO}

No estado do Acre, em 2017, notificou-se 209 casos de indivíduos acometidos por leptospirose conforme apresentado na figura 1. Verificou-se que dos 209 casos notificados, a maior parcela dos casos foi no sexo masculino (66,5\%). Realidade também encontrada nos estudos de Baracho, Lima e Costa (2017), Oliveira et al. (2016), Basso e Righi (2015), Pereira et al. (2014) e Sampaio et al. (2011) que revelam que o perfil epidemiológico da doença em várias cidades do país é de prevalência no sexo masculino. Acredita-se que a prevalência em homens adultos muito provavelmente é decorrente do fato de estarem mais expostos, por atuarem em profissões de salvamento de possíveis vítimas de temporais e enchentes (SOARES et al., 2009; SILVA; MOREIRA, 2013). E também por serem os que mais exercem atividades de risco, como coleta de lixo urbano, limpeza de bueiros, instalação e manutenção de rede de esgoto, controle de roedores, entre outras atividades de risco (BUZZAR, 2011).

$\mathrm{Na}$ figura 2, foram trazidas informações quanto a faixa etária dos indivíduos, independentemente do sexo. Revelou-se que as maiores proporções em relação à faixa 
etária dos pacientes foram de 20-39 anos (38,8\%) e 40-59 anos (32\%). Resultados consoantes com os encontrados em Oliveira et al. (2016) na baixada fluminense, estado do Rio de Janeiro de 20-39 anos (34,3\%) e 40-59 anos (28,1\%), Basso e Righi (2015) em Porto Alegre, estado do Rio Grande do Sul de 20-59 anos (75,5\%) e Baracho, Lima e costa (2017) no estado de Pernambuco de 20-39 anos (38,8\%) e 40-59 anos (27,5\%). Esses dados permitem inferir que a leptospirose acomete em sua grande maioria a população na faixa etária economicamente produtiva no país (BRASIL, 2014).

$\mathrm{Na}$ figura 3, o nível de escolaridade dos pacientes evidenciou que os maiores percentuais dos casos foram em indivíduos com o ensino fundamental incompleto $(35,9 \%)$ e nível escolar ignorado/branco (19,1\%). Além disso, observou-se que pacientes com educação superior incompleta ou completa apresentaram (2\%) dos casos notificados. Este achado pode estar relacionado com o nível de escolaridade da população em geral no estado, em que a população de 25 anos ou mais em 2010, mostrou que 21,29\% eram analfabetos, 43,98\% tinham ensino fundamental completo, 31,56\% possuíam o ensino médio completo e $8,98 \%$ o superior completo (ATLAS DO DESENVOLVIMENTO HUMANO NO BRASIL, 2010). Dados semelhantes ainda foram encontrados nos trabalhos de Basso e Righi (2015) em Porto Alegre-RS, 45\% tinham o ensino fundamental incompleto e no de Baracho, Lima e costa (2017) no estado de Pernambuco-PE, apenas 1,2\% dos afetados possuíam educação superior.

Nesse sentido, pode-se observar que o baixo nível de escolaridade também é uma variável que contribui para a ocorrência da leptospirose e de outras doenças, devido à adoção de práticas prejudiciais, como o acúmulo de materiais recicláveis em condições inadequadas (MESQUITA et al., 2016), falta de compreensão quanto aos meios de prevenção e transmissão da doença, entre outras (CARVALHO et al., 2017). Por outro lado, indivíduos com maior nível de escolaridade tendem a ter atitudes que diminuem as chances de contrair leptospirose, como não acumular entulhos, beber água tratada, andar calçado e evitar tomar banho em inundações, rios e igarapés contaminados por esgoto.

Na tabela 1 em relação à classificação da zona de residência e os municípios onde houve notificação, verificou-se que os casos ocorreram principalmente na zona urbana (69,9\%) e os municípios com maior proporção de casos foram Rio Branco (44,5\%) e Cruzeiro do Sul-Acre (37,8\%). Realidade confirmada também nos estudos de Pereira et al. (2016), Pereira et al. (2014), Sampaio et al. (2011) e Souza et al. (2011) em que a ocorrência da leptospirose é de predominância na zona urbana. Quanto aos maiores percentuais da 
doença terem ocorrido nos dois principais municípios do estado (Rio Branco e Cruzeiro do Sul), destaca-se que ambos são referência para a atenção à saúde da população do Acre, podendo inclusive receber pacientes/casos de outras cidades circunvizinhas. Além disso, acredita-se também que possam estar relacionados ao crescimento demográficopopulacional desorganizado (ALVES, 2008), infraestrutura sanitária deficiente (CLAZER et al., 2015), saneamento básico que ainda não atende a todos (FONSECA et al., 2011), além da questão das cidades terem crescido às margens do Rio Acre e Rio Juruá e seus afluentes que em tempos de chuvas transbordam e inundam vários pontos das cidades; situação que favorece para a disseminação da doença pela mistura de água com esgoto (SILVA, 2013).

Na tabela 2, quanto à evolução e o desfecho clínico dos pacientes no estado do Acre, constatou-se uma baixa letalidade da leptospirose em que (96,1\%) dos casos obtiveram cura, $(2,4 \%)$ desfecho ignorado/branco e apenas (1\%) foram a óbito pela doença. Esta baixa letalidade deve-se, provavelmente, ao diagnóstico oportuno e tratamento adequado, bem como aos poucos casos que podem ter progredido para condições graves, culminando em óbito pelo agravo notificado. Um estudo de Calado et al. (2017) sobre a leptospirose no norte do Brasil, no período de 2012 a 2015, mostrou uma redução no número de mortes pela doença na região norte, com uma proporção de cura de 93,37\%. Diferentemente dos dados encontrados nas regiões Nordeste $76,70 \%$, Sudeste $75,82 \%$ e Centro-Oeste $77,45 \%$ do mesmo estudo, demonstrando uma maior deficiência no manejo da doença do que na região norte do país.

Neste sentido, o perfil epidemiológico dos pacientes com diagnóstico confirmado de leptospirose no Estado do Acre, em 2017, demonstra que quando os pacientes são diagnosticados em tempo hábil e submetidos a tratamento especializado, as chances de cura são elevadas. No entanto, há necessidade de adoção de medidas preventivas em saúde pública, uma vez que o número de casos ocorridos foi alta, o que não pode ser aceitável, já que a leptospirose é uma doença passível de prevenção.

\section{CONCLUSÃO}

A leptospirose é uma doença endêmica no Brasil, decorrente de questões ambientais, infraestrutura urbana precária e desigualdade socioespacial. No estado do Acre, o estudo 
dos casos de leptospirose mostrou relação com os mesmos fatores que favorecem o desenvolvimento da patologia em nível nacional, mas também foi perceptível a baixa letalidade da doença, muito provavelmente devido ao diagnóstico e tratamento oportunizado. 0 sexo masculino foi o mais acometido pela doença, com faixa etária entre 20 a 39 anos e nível fundamental incompleto; a maior incidência de infecção ocorreu na área urbana nas duas principais cidades: Rio Branco e Cruzeiro do Sul.

No que diz respeito à profilaxia da leptospirose, é necessário adotar e aplicar atitudes pessoais de prevenção (educação em saúde), e que a administração pública estadual tem o dever de proporcionar ao indivíduo saneamento básico e infraestrutura adequada.

Por fim, ressalta-se a importância de estudos futuros envolvendo esse tema, principalmente quanto à análise e padrão de sazonalidade da doença, haja vista a sua importância na área de Saúde Coletiva em nosso país.

\section{REFERÊNCIAS}

ALVES, Lucimara Aparecida. Soroepidemiologia da leptospirose em mulheres gestantes do município de Londrina - PR. 2008. 43f. Dissertação (Mestrado em Ciência Animal) - Universidade Estadual de Londrina, Londrina, PR. Disponível em: < http://www.bibliotecadigital.uel.br/document/?code=vtls000135392>. Acesso em: 09 jun. 2018.

ATLAS DO DESENVOLVIMENTO HUMANO NO BRASIL. Acre. Disponível em: < http://www.atlasbrasil.org.br/2013/pt/perfil_uf/acre/\#educacao>. Acesso em: 15 mar. 2019.

AVILA-PIRES, Fernando Dias de. Leptospirose e enchentes: uma falsa correlação?. Revista de Patologia Tropical, v. 35, n. 3, p. 199-204, 2006. Disponível em: < https://www.revistas.ufg.br/iptsp/article/view/1880>. Acesso em: 14 jun. 2018.

BARACHO, Juliana Mendes; LIMA, Nadiely de Barros; COSTA, Ana Paula Rocha. Incidência de casos de Leptospirose Humana em Pernambuco: uma análise dos dados epidemiológicos de 2015. Caderno de Graduação-Ciências Biológicas e da Saúde-FACIPE, v. 3, n. 2, p. 19, 2017. Disponível em: https://periodicos.set.edu.br/index.php/facipesaude/article/view/5153/2545>. Acesso em: 06 jun. 2018.

BASSO, Luís Alberto; RIGHI, Eléia. Casos de hepatite e leptospirose no município de Porto Alegre-RS entre os anos de 2007 a 2011. Hygeia: Revista Brasileira de Geografia Medica e da Saúde, v. 11, n. 20, p. 66, 2015. Disponível em:< https://search.proquest.com/openview/220f784dfa9c38fb13046e8d1331a617/1?pqorigsite $=$ gscholar\&cbl=2030864>. Acesso em: 06 jun. 2018.

BRASIL. Ministério da Saúde. Departamento de informática do Sistema Único de Saúde-DATASUS. Leptospirose: Secção Acre. Disponível em: $<$ http://tabnet.datasus.gov.br/cgi/deftohtm.exe?sinannet/cnv/leptoac.def>. Acesso em: 02 jun. 2018.

BRASIL. Ministério da Saúde. Secretária de vigilância em Saúde. Guia de Leptospirose: Diagnósticos e manejo clínico. Ministério da Saúde. 2009. Disponível em: $<$ https://www.nescon.medicina.ufmg.br/biblioteca/imagem/leptospirose-manejo-clinico.pdf >. Acesso em: 17 jun. 2018. 
Perfil epidemiológico de pacientes acometidos por

leptospirose em um estado brasileiro na Amazônia Ocidental

BRASIL. Ministério da Saúde. Secretaria de Vigilância em Saúde. Leptospirose: diagnóstico e manejo clínico. Brasília: Ministério da $\quad$ Saúde, 2014. $\quad$ Disponível em: 2014.pdf> . Acesso em: 08 jun. 2018.

BRASIL. Ministério da Saúde. Leptospirose: análise dos dados epidemiológicos de 2010 a 2014. 2016. Disponível em:< http://portalarquivos2.saude.gov.br/images/pdf/2016/maio/20/Informe-epidemiol--gicoleptospirose.pdf>. Acesso em: 22 mar. 2019.

BRASIL. Ministério da Saúde. Leptospirose. 2017a. Disponível em:< http://portalms.saude.gov.br/saude-de-az/leptospirose>. Acesso em: 16 jun. 2018.

BRASIL. Ministério da Saúde. Secretaria de Vigilância em Saúde. Guia de Vigilância em Saúde. 2. Edição. Brasília: Ministério da Saúde, 2017b. Disponível em: <http://portalarquivos.saude.gov.br/images/pdf/2017/outubro/06/Volume-Unico-2017.pdf>. Acesso em: 09 jun. 2018.

BORGHI, Ana Flávia Rodrigues; QUEIROZ, Silvio José de. Distribuição da leptospirose humana no Brasil. Revista Estudos Vida e Saúde, v. 44, n. 1, p. 115-123, 2017. Disponível em: < http://seer.pucgoias.edu.br/index.php/estudos/article/download/5958/3323>. Acesso em: 22 mar. 2019.

BUZZAR, Márcia Regina. Perfil epidemiológico da leptospirose no estado de São Paulo no período de 2007 a 2010. In: Anais da $\mathbf{1}^{\mathrm{a}}$ Conferência Internacional em Epidemiologia. 2011. Disponível em: < ftp://ftp.cve.saude.sp.gov.br/doc_tec/Zoo/lepto11_poster_abrasco.pdf>. Acesso em: 07 jun. 2018.

CALADO, Enoque Júnior da Rocha; OLIVEIRA, Vitória de Souza; DIAS, Fellipe Camargo Ferreira; LOPES, Aline Barbosa; OLIVEIRA, Amanda Amâncio; SANTANA, Victor Mateus Xavier; GUSMÃO, Kamile Eller; LOBO, Pedro Henrique Procópio; LIBERATO, Aline Almeida; GUEDES, Virgílio Ribeiro. Leptospirose na região norte do Brasil: uma revisão da literatura e perfil epidemiológico comparativo. Revista de Patologia do Tocantins, v. 4, n. 2, p. 65-71, 2017. Disponível em: < https://sistemas.uft.edu.br/periodicos/index.php/patologia/article/view/3759>. Acesso em: 15 mar. 2019.

CARVALHO, Cristiane Borba Campos; GOMES, Marina Letícia Coelho; SANTOS, Cristiane Lopes; RABELlO, Renata dos Santos; THOMÉ, Sandra Maria Gomes. Leptospirose humana no estado do Rio de Janeiro: análise espaço-temporal e perfil dos casos confirmados no período de 2007 a 2014. Academus Revista Científica da Saúde, [S.l.], v. 2, n. 3, dez. 2017. ISSN 1806-9495. Disponível em: < https://smsrio.org/revista/index.php/reva/article/view/343> . Acesso em: 08 jun. 2018.

CASTRO, Jacqueline Ribeiro; SALABERRY, Sandra Renata Sampaio; SOUZA, Mariana Assunção; LIMA-RIBEIRO, Anna Monteiro Correia. Sorovares de Leptospira spp. predominantes em exames sorológicos de caninos e humanos no município de Uberlândia, Estado de Minas Gerais. Rev Soc Bras Med Trop, v. 44, n. 2, p. 217-222, 2010. Disponível em: < http://www.scielo.br/pdf/rsbmt/2011nahead/aop12-11.pdf >. Acesso em: 13 jun. 2018.

CHAIBLICH, Juliana Valentim; LIMA, Maria Luciene da Silva; OLIVEIRA, Raiane Fontes; MONKEN, Maurício; PENNA, Maria Lucia Fernandes. Estudo espacial de riscos à leptospirose no município do Rio de Janeiro (RJ). Saúde em Debate, v. 41, p. 225-240, 2017. Disponível em: <https://www.scielosp.org/pdf/sdeb/2017.v41nspe2/225-240/pt> . Acesso em: 18 jun. 2018.

CLAZER, Marília. Leptospirose e seu aspecto ocupacional - revisão de literatura. Arquivos de Ciências Veterinária e Zoologia-UNIPAR, Umuarama, v.18, n. 3, p. 191-198, jul./set. 2015. Disponível em: < http://revistas.unipar.br/index.php/veterinaria/article/view/5541>. Acesso em: 09 jun. 2018.

COSTA, Federico. Morbidade Global e Mortalidade da Leptospirose: Uma Revisão Sistemática. PLoS Negl Trop Dis , v. 9, p. 1-19, 2015. Disponível em: <https://doi.org/10.1371/journal.pntd.0003898>. Acesso em: 15 mar. 2019.

DAHER, Elizabeth De Francesco; BRUNETTA, Denise Menezes; SILVA JÚNIOR, Geraldo Bezerra; PUSTER, Rainardo Antoni; PATROCíNIO, Régia Maria do Socorro Vidal. Características clínicas e epidemiológicas da 
leptospirose e análise retrospectiva dos casos ocorridos no Ceará. Revista Brasileira de Medicina, 2003. Disponível em: <http://www.moreirajr.com.br/revistas.asp?id_materia=2986\&fase=imprime>. Acesso em: 16 jun. 2018.

FONSECA, Zuliete Aliona Araujo de Souza; BEZERRA, Rosivaldo Quirino; MOURA, Edinaidy Suianny Rocha; SOUSA, Êlika Suzianny; OLIVEIRA, Gleidson Benevides. Roedores como um problema de saúde pública: experiência de programa de controle. Publicações em Medicina Veterinária e Zootecnia-PUBVET, Londrina, V. 5, N. 36, Ed. 183, 2011. Disponível em: < http://www.pubvet.com.br/artigo/2074/p-styletextalign-justify-aligncenterstrongroedores-como-um-problema-de-sauacutede-puacuteblica-experiecircncia-deprograma-de-controlestrongp>. Acesso em: 09 jun. 2018.

FONZAR, Udelysses Janete Veltrini. Análise geográfica da ocorrência da leptospirose em humanos e em cães na cidade de Maringá, Paraná, Brasil. 2010. Disponível em: < https://repositorio.unesp.br/handle/11449/101470 >. Acesso em: 14 jun. 2018.

GOVERNO DO ESTADO DA BAHIA. Secretaria da Saúde. Diretoria de Vigilância Epidemiológica/ Superintendência de Vigilância em Saúde [DIVEP -SUVISA]. Boletim Epidemiológico de Leptospirose Bahia 2017. Bahia, n. 2, Dez., 2017. Disponível em: <http://www.saude.ba.gov.br/wpcontent/uploads/2017/11/Boletim-Epidemiol\%C3\%B3gico-de-Leptospirose-Bahia-2017-n2.pdf>. Acesso em: 21 mar. 2019.

MESQUITA, Marilise Oliveira; TREVILATO, Graziella Chaves; SARAIVA, Luiza de Holleben; SCHONS, Michelle da Silva; GARCIA, Maria Isabel Ferreira. Material de educação ambiental como estratégia de prevenção da leptospirose para uma comunidade urbana reassentada. Cadernos saúde coletiva (Rio de Janeiro). Vol. 24, n. 1 , p. 77-83, 2016. Disponível em: < https://www.lume.ufrgs.br/handle/10183/142305> . Acesso em: 08 jun. 2018.

OLIVEIRA, Heloiza Helena; RODRIGUES, Marcos Aurélio Machado; SANTOS, Iramar Santana; FRANCISCHETTI, Cesar Nascimento. Perfil epidemiológico e socioeconômico da ocorrência de casos de leptospirose em municípios da Baixada Fluminense, Rio de Janeiro, Brasil. Enciclopédia Biosfera, v. 13, n. 23, p. 1479-91, 2016. Disponível

< http://www.conhecer.org.br/enciclop/2016a/ciencias\%20da\%20saude/perfil\%20epidemiologico.pdf>.

Acesso em: 06 jun. 2018.

PEREIRA, Bruna Fernandes; BASSANI, Debora Cristina Haack; CHAVES, Jéssica; TABILE, Patrícia Micheli; KRUMMENAUER, Eliane Carlosso; CARNEIRO, Marcelo. Perfil Clínico da Leptospirose em Santa Cruz do Sul (RS): Revisão Dos Anos De 2012 E 2013. Blucher Medical Proceedings, v. 1, n. 5, p. 39-39, 2014. Disponível em: $<$ http://www.proceedings.blucher.com.br/article-details/perfil-clnico-da-leptospirose-em-santa-cruz-do-sulrs-reviso-dos-anos-de-2012-e-2013-13355> . Acesso em: 06 jun. 2018.

PEREIRA, Simone Valeria Costa; OLIVEIRA, Stefan Vilges; FONSECA, Lidsy Ximenes; PINNA, Fernanda Voietta; CALDAS, Eduardo Pacheco. Boletim epidemiológico da leptospirose no Brasil no período de 2010 a 2014. Disponível em: <http://www.sbmt.org.br/medtrop2016/wp-content/uploads/2016/11/10749-Boletimepidemiolo\%CC\%81gico-da-leptospirose-no-Brasil-no-peri\%CC\%81odo-de-2010-a-2014..pdf>. Acesso em: 08 jun. 2018.

SAMPAIO, Giorge Pereira; WANDERLEY, Mariane Rodrigues; CASSEB, Giovanni Bady; NEGREIROS, Marcelus Antonio Motta Prado. Descrição epidemiológica dos casos de leptospirose em hospital terciário de Rio Branco. Revista Brasileira Clínica Médica, v. 9, n. 5, p. 338-343, 2011. Disponível em: < http://files.bvs.br/upload/s/1679-1010/2011/v9n5/a2246.pdf>. Acesso em: 06 jun. 2018.

SILVA, Alice Martins. Leptospirose no Distrito Federal: Perfil epidemiológico e caracterização dos casos dos prováveis locais de infecção dos casos humanos autóctones confirmados em 2011 e 2012. 2013. Monografia (Conclusão do curso de Medicina Veterinária) - Faculdade de Agronomia e Medicina Veterinária Universidade de Brasília, Brasília, DF. Disponível em: < http://bdm.unb.br/handle/10483/4782>. Acesso em: 09 jun. 2018. 
Perfil epidemiológico de pacientes acometidos por

leptospirose em um estado brasileiro na Amazônia Ocidental

SILVA, Patricia Lizandra; MOREIRA, Somine Magela. Leptospirose: fatores ambientais que favorecem a sua ocorrência em humanos. Acervo da iniciação científica, n. 1, 2013. Disponível em: http://www3.izabelahendrix.edu.br/ojs/index.php/aic/article/view/413. Acesso em: 07 jun. 2018.

SIMÕES, Luciana Senna; SASAHARA, Tais Harumi de Castro; FAVARON, Phelipe Oliveira; MIGLINO, Maria Angelica. Leptospirose-Revisão. PUBVET, v. 10, p. 111-189, 2016. Disponível em: < http://www.pubvet.com.br/uploads/ac2bca4566fbc69dc78fdfb5bbd21be4.pdf>. Acesso em: 13 jun. 2018.

SOARES, Tatiana Spinelli Martins; LATORRE, Maria do Rosário Dias de Oliveira; LAPORTA, Gabriel Zorello; BUZZAR, Márcia Regina. Análise espacial e sazonal da leptospirose no município de São Paulo, SP, 1998 a 2006. Revista de Saúde Pública, v. 44, n. 2, p. 283-291, 2009. Disponível em: < https://www.scielosp.org/scielo.php?pid=S0034-89102010000200008\&script=sci_abstract\&tlng=es >. Acesso em: 07 jun. 2018.

SOUZA, Vânia Rodrigues de. LEPTOSPIROSE: Aspectos epidemiológicos, clínicos e laboratoriais. 2011. Disponível em:

http://www.pergamum.univale.br/pergamum/tcc/Leptospiroseaspectosepidemiologicosclinicoselaboratoriai s.pdf>. Acesso em: 14 jun. 2018.

SOUZA, Verena Maria Mendes; ARSKY, Maria de Lourdes Nobre Simões; CASTRO, André Peres Barbosa; ARAUJO, Wildo Navegantes. Anos potenciais de vida perdidos e custos hospitalares da leptospirose no Brasil. Revista de Saúde Pública, v. 45, p. 1001-1008, 2011. Disponível em: < https://www.scielosp.org/article/rsp/2011.v45n6/1001-1008/pt/>. Acesso em: 06 jun. 2018.

SUPERINTENDÊNCIA DA ZONA FRANCA DE MANAUS-SUFRAMA. Amazônia Ocidental. Disponível em: <http://site.suframa.gov.br/assuntos/amazonia-ocidental >. Acesso em: 02 jun. 2018.

\section{(cc) BY}

Este trabalho está licenciado com uma Licença Creative Commons - Atribuição 4.0 Internacional. 